\title{
Expression of progenitor cell/immature neuron markers does not present definitive evidence for adult neurogenesis
}

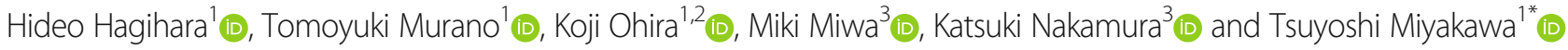

\begin{abstract}
It is agreed upon that adult hippocampal neurogenesis (AHN) occurs in the dentate gyrus (DG) in rodents. However, the existence of AHN in humans, particularly in elderly individuals, remains to be determined. Recently, several studies reported that neural progenitor cells, neuroblasts, and immature neurons were detected in the hippocampus of elderly humans, based on the expressions of putative markers for these cells, claiming that this provides evidence of the persistence of AHN in humans. Herein, we briefly overview the phenomenon that we call "dematuration," in which mature neurons dedifferentiate to a pseudo-immature status and re-express the molecular markers of neural progenitor cells and immature neurons. Various conditions can easily induce dematuration, such as inflammation and hyper-excitation of neurons, and therefore, the markers for neural progenitor cells and immature neurons may not necessarily serve as markers for AHN. Thus, the aforementioned studies have not presented definitive evidence for the persistence of hippocampal neurogenesis throughout adult life in humans, and we would like to emphasize that those markers should be used cautiously when presented as evidence for AHN. Increasing AHN has been considered as a therapeutic target for Alzheimer's disease (AD); however, given that immature neuronal markers can be re-expressed in mature adult neurons, independent of $A H N$, in various disease conditions including $A D$, strategies to increase the expression of these markers in the DG may be ineffective or may worsen the symptoms of such diseases.
\end{abstract}

Keywords: Hippocampus, Dentate gyrus, Granule cells, Dematuration, Adult neurogenesis

\section{Introduction}

There is an agreement that new neurons are generated in the dentate gyrus (DG) of hippocampus in rodents and non-human primates during adulthood, but it remains to be determined whether the adult hippocampal neurogenesis (AHN) commonly occurs in humans throughout aging. Recently, Moreno-Jiménez and colleagues reported that there are a number of neurons in the human DG that express immature neuron markers, such as doublecortin (DCX) and calretinin (CR), which are believed to be "reliable" neurogenic markers (See Additional file 1: Table S1), claiming that this provides evidence for the persistence of AHN in aged humans [1]. Other studies by Boldrini et al. [2] and Tobin et al.

\footnotetext{
*Correspondence: miyakawa@fujita-hu.ac.jp

'Division of Systems Medical Science, Institute for Comprehensive Medical

Science, Fujita Health University, Toyoake, Aichi 470-1192, Japan

Full list of author information is available at the end of the article
}

[3] that used similar markers also arrived at the same conclusions. Moreno-Jiménez et al. reported decreased expression of immature neuronal markers in patients with Alzheimer's disease (AD) compared with neurologically healthy subjects [1], which was not replicated by Tobin et al. [3]. However, these immature neuronal markers can be re-expressed in pre-existing mature neurons by neural excitation and drug administration, and their expression can be independent of adult neurogenesis (Fig. 1). Therefore, data shown by the abovementioned studies may not serve as definitive evidence of the existence of human adult neurogenesis. Herein, we briefly review the previously published literature that have demonstrated that immature neuronal markers, such as DCX and CR, can be re-expressed in preexisting mature neurons via a process that we refer to as dematuration. We note the possibility that molecular expression pattern indicative of immature neurons might

(c) The Author(s). 2019 Open Access This article is distributed under the terms of the Creative Commons Attribution 4.0 International License (http://creativecommons.org/licenses/by/4.0/), which permits unrestricted use, distribution, and 


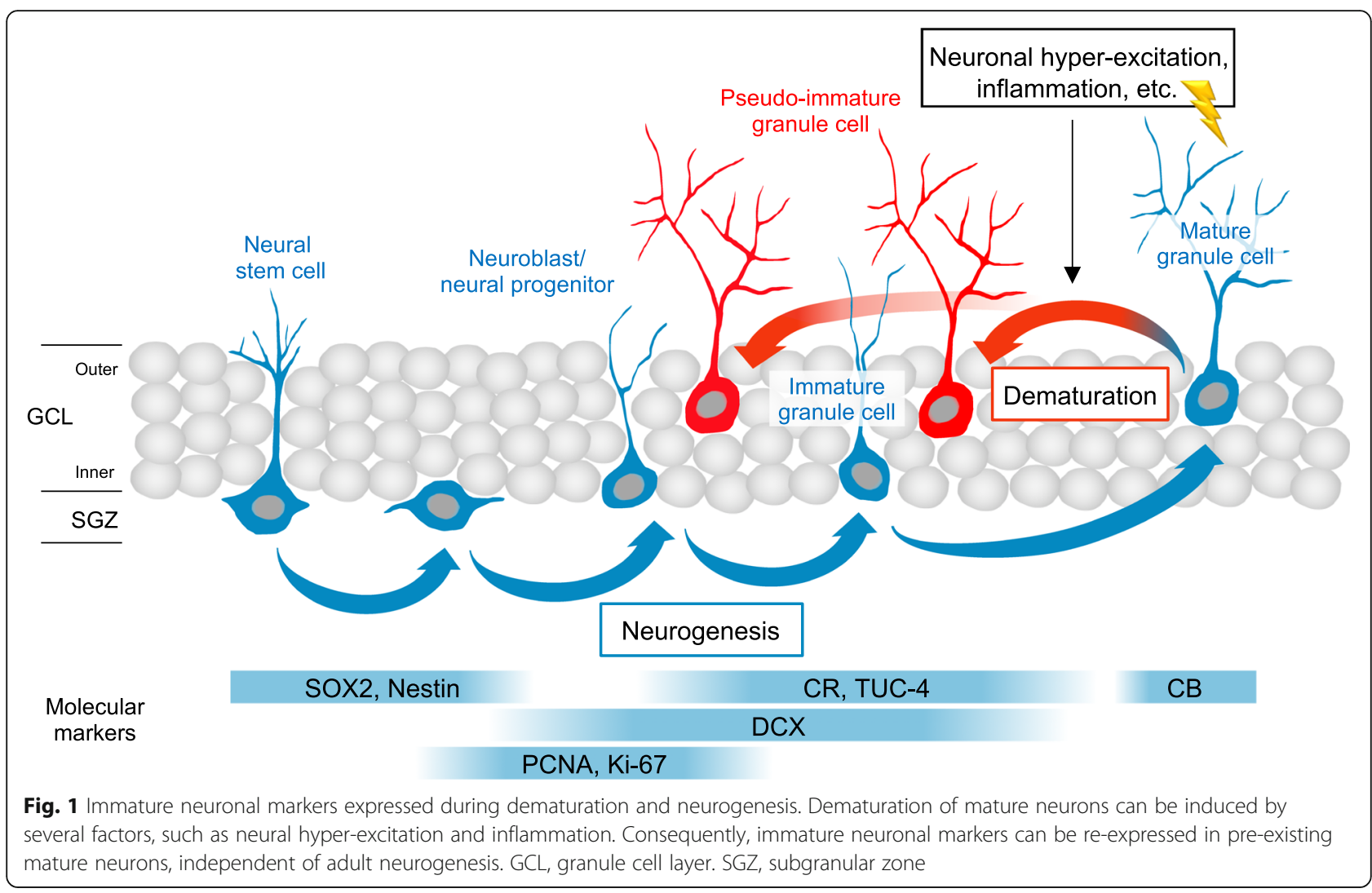

occur in the DG of patients with AD by demonstrating the similarity between the molecular expression patterns observed in the DG of patients with $\mathrm{AD}$ and that of mice with an "immature dentate gyrus" phenotype.

\section{Re-expression of cell cycle/progenitor markers in mature neurons by cell cycle re-entry}

A series of studies have shown ectopic expression of stem cell / cell cycle markers, such as Sox2, PCNA, and $\mathrm{Ki}-67$, in mature postmitotic neurons, including granule cells (GCs), in the hippocampal DG in the brains of patients with $\mathrm{AD}$ and other neurodegenerative disorders $[4,5]$ (for references, see Additional file 1: Table S2). It has been demonstrated that neuronal death is preceded by re-expression of cell cycle markers in mature neurons and that various insults, including toxic concentrations of amyloid-ßand hyper-excitation of neurons induced by kainic acid, can lead to the expression of these markers [4]. This cell cycle re-entry of postmitotic neurons is postulated as an additional or alternative mechanism contributing to the symptoms of AD [6]. Therefore, the expression of these cell cycle markers may not necessarily indicate AHN. Although cells expressing these markers were seen in the subgranular zone (a wellknown neurogenic region) and the middle and outer parts of the granule cell layer (GCL; where mature granule cells are typically located) [2, 3], a significant proportion of these expressions, albeit not all, especially within the GCL, could be contributed by the re-entry of mature GCs into the cell cycle, rather than due to AHN.

Supporting evidence for dematuration of mature neurons There are several lines of evidence supporting the existence of dematuration in particular types of neurons [7-13]. Our study, along with other studies, has demonstrated that the maturation of GCs in the DG is a dynamic and reversible process, involving changes in their molecular expression patterns and electrophysical properties [7, 8] (Fig. 1; Additional file 1: Table S2). Chronic treatment with the antidepressant, fluoxetine (FLX), causes the reversal of mature adult GCs back to a pseudo-immature status [7]. Mice were injected with bromodeoxyuridine (BrdU), which gets incorporated into the newly synthesized DNA strand of proliferating cells and can be used as a cytogenetic marker, at neonatal period (postnatal days 1-3), and thereafter, treated with FLX or vehicle at adulthood (at 9 weeks of age) for 45 weeks. It was found that in the adult DG, BrdU-positive $\left(\mathrm{BrdU}^{+}\right)$cells, i.e., putative mature GCs, expressed calbindin (CB), a marker of mature GCs, in the vehicle-treated control mice. However, many BrdU ${ }^{+}$cells lacked $\mathrm{CB}$ expression in FLX-treated animals, indicating that the mature neurons lost CB expression as a result of FLX treatment [7]. Newly generated immature GCs in adults are located in the inner part of the GCL or the subgranular zone, as assessed by 
BrdU labeling. However, the reduction in $\mathrm{CB}$ and the overexpression of DCX and CR, which are also immature GC markers, can also be found in the middle or outer layer of the GCL in FLX-treated animals [7], supporting the idea that "dematuration," the process by which mature GCs return to a pseudo-immature state, can be induced in GCs by FLX treatment. CB, along with more than 380 mature marker genes and 150 immature marker genes, were down or up-regulated in the DG of the mice that received chronic treatment with FLX, which cannot be explained solely by an increase in AHN [8].

Dematuration of GCs can also result from epileptic seizures induced by pilocarpine [13] and electroconvulsive shocks [14] in rodents, as assessed by CB expression, genome-wide gene expression patterns, and/or the electrophysiological properties of GCs. Mice with mutant synaptosomal-associated protein 25 (SNAP25) exhibit epileptic seizures occasionally and have the socalled "immature dentate gyrus" phenotype, which can be treated by preventing epileptic seizures via the administration of an anti-epileptic drug [15]. Decreased expression of $\mathrm{CB}$ has also been observed in the $\mathrm{DG}$ of patients with epilepsy [16, 17], and the expression of $C B$ is also decreased in the DG of AD mouse models and $\mathrm{AD}$ patients $[18,19]$. Considering that epileptic seizures have been observed in patients with $\mathrm{AD}$ [20], and seizure frequency is negatively correlated with $\mathrm{CB}$ levels in the DG of AD model mice [18], this decreased expression of $\mathrm{CB}$ in the DG may be a result of the epileptic seizures. Gene expression signatures of neuronal hyper-excitation and immaturity have been found post-mortem in the brains of patients with AD, schizophrenia, and amyotrophic lateral sclerosis [21]. These findings indicate that hyper-excitation of neurons can cause dematuration in the brains of rodents and, probably humans, including AD patients.

\section{AHN and dematuration are separate phenomena}

AHN and dematuration are separate phenomena, since dematuration in the DG is accompanied by increased, decreased, or unchanged AHN. Increased AHN is a well-established and intensely investigated phenomenon, including in mice that were chronically treated with FLX $[22,23]$. Increased adult neurogenesis was seen in a few different kinds of mice with an "immature dentate gyrus" phenotype [11, 14, 24]. On the contrary, in SNAP25 mutant mice with an "immature dentate gyrus" phenotype, the AHN, as assessed by BrdU labeling, was almost absent [15]. The relative position of $\mathrm{DCX}^{+}$cells shifted from the subgranular zone to the middle or outer GCL in these mutant mice. However, it is unlikely that DCX expression in the middle or outer GCL is due to AHN, because BrdU staining was mostly restricted to the subgranular zone in these mutant mice, as found in the wild-type mice.

In the DG of common marmosets, the expression levels of immature GC markers (e.g., DCX and CR) were increased without any increase in adult neurogenesis in response to chronic antidepressant treatment, as assessed by BrdU staining, which is a more robust cytogenesis marker [10]. It should be noted that a number of $\mathrm{DCX}^{+}$cells were observed in the middle and outer GCL, while $\mathrm{BrdU}^{+}$cells were only observed in or close to the subgranular zone, exemplifying that adult neurogenesis and dematuration were separate phenomena, and demonstrating that the expression of immature GC markers does not necessarily serve as evidence for AHN in nonhuman primates. AHN is thought to occur in or close to the subgranular zone, which was also seen through BrdU staining of the DGs of non-human primates [10]. Figure 2 in the paper by Tobin et al. [3] shows that $\mathrm{DCX}^{+}$cells are located not only around the subgranular zone, but also in the middle and outer parts of the GCL, where mature GCs are typically located, in elderly humans and $\mathrm{AD}$ patients, raising the possibility that at least some of the $\mathrm{DCX}^{+}$cells are GCs that underwent dematuration, rather than being newly generated. Identifying the positions of cells that express immature GC markers within the GCL, either near to or far from the subgranular zone, might be one way to discriminate whether those cells are derived from neurogenesis or dematuration. Considering that both mature astrocytes and postmitotic neurons that express DCX have been observed in the cortex [25, 26], the possibility of the existence of cell types, other than GCs that have undergone dematuration, that express immature GC markers should also be taken into account. In addition, if neurogenic markers that are expressed independent of dematuration are identified in future studies, they could provide a useful means to discriminate these two phenomena.

Ectopic expression of immature neuronal markers can be found in the middle and outer GCL of patients with epilepsy, which presents the possibility that these neurons may also be formed by adult neurogenesis [27]. If this mechanism also existed in FLX-treated marmosets and SNAP25 mutant mice, upon BrdU incorporation assay, $\mathrm{DCX}^{+}$or $\mathrm{CR}^{+} \mathrm{GCs}$ located in the middle and outer GCL should have been labeled with BrdU, which should have been incorporated in the process of adult neurogenesis. However, the positions of the $\mathrm{DCX}^{+}$or $\mathrm{CR}^{+}$cells and $\mathrm{BrdU}^{+}$cells differed from each other within the GCL in FLX-treated marmosets and SNAP25 mutant mice; a number of $\mathrm{DCX}^{+}$or $\mathrm{CR}^{+}$cells were located in the middle and outer regions of the GCL, whereas almost all of the $\mathrm{BrdU}^{+}$cells were confined to the subgranular layer in these animals. Thus, the expression of immature neuronal markers in the middle and 
outer GCL may not be attributed to adult neurogenesis, but rather to the dematuration of mature neurons.

Re-expression of cell cycle and immature markers in mature GC neurons does not completely disprove the existence of human AHN. Strong evidence for AHN in humans has been provided by a birth-dating study of dividing precursor cells using ${ }^{14} \mathrm{C}$ incorporation into synthesized DNA, which did not rely on the detection of molecular markers [28]. It has been suggested, however, that the extent of AHN may be overestimated in immunohistochemical studies of immature marker expression in comparison to ${ }^{14} \mathrm{C}$ birth-dating data [29]. This potential overestimation may be due to that GCs that underwent dematuration were also considered as immature neurons derived from $\mathrm{AHN}$ in immunohistochemical studies.

\section{Dematuration of mature neurons as a hallmark of AD and aging}

If cells dedifferentiate in diseased or damaged conditions, such as in $\mathrm{AD}$, the proportion of cells expressing immature neuronal markers should be greater in the brains of patients with $\mathrm{AD}$. Indeed, many previous reports show increased expression of immature GC markers in the brains of patients with $\mathrm{AD}$ [30-32] and other neurodegenerative disorders [33], whereas Moreno-Jiménez et al. reported decreased expression of immature neuronal markers in the DG of patients with AD. Also, Tobin et al. found no statistically significant decrease in the number of cells expressing immature GC markers in patients with $\mathrm{AD}$ as compared to control individuals [3]. Therefore, one might argue that it is inconsistent with the hypothesis that dematuration or cell cycle re-entry is increased in patients with AD. In fact, however, in Figure 2 and Figure 4 of the report by Tobin et al., patients with $\mathrm{AD}$ showed the highest level of $\mathrm{DCX}^{+}$or $\mathrm{DCX}^{+} / \mathrm{PCNA}^{+}$cell numbers [3]. In addition, considering that 1 ) those markers are overexpressed in many brain regions other than the DG in patients with $\mathrm{AD}$, and 2) neuronal loss in the hippocampus of patients with AD happens mostly in the CA1 area and not much in the DG [34-36], these cell cycle / immature neuronal markers need not be overexpressed exclusively in the DG of patients with AD (the most affected brain region may differ among individual patients). Moreover, a decrease of DCX expression does not necessarily mean reduced dematuration in these patients. As mentioned above, SNAP25 mutant mice exhibit severe dematuration phenotypes in the DG with regard to CB expression, genome-wide gene expression patterns, and electrophysiological properties, whereas DCX expression is significantly decreased in these mice [15]. Thus, the potential increase of dematuration in patients with $\mathrm{AD}$ may not be inconsistent with decreased DCX expression.
Further, we would like to mention that dematuration can occur even in seemingly normal neurons. Differentiated neurons can re-express cell cycle markers by neuronal hyper-excitation $[37,38]$. It is easy to imagine that such neuronal hyper-excitation can occur in apparently healthy aged individuals. Interestingly, there is a group of putative marker genes for immature neurons that show upregulation during brain development, and tend to exhibit peak expression at approximately 26 years of age and gradually decrease with aging [39]. This suggests that a dematuration-like phenomenon, demonstrated by gene expression patterns, may occur in healthy, aging individuals.

\section{Conclusions}

Given that cell cycle and immature GC markers can be re-expressed in mature adult neurons, independent of AHN in rodents and primates, it is quite possible that at least a part of the expression of those markers in the human DG may not be due to AHN, but due to the cell cycle re-entry /dematuration of old mature neurons. Reexpression of cell cycle and immature markers in mature GC neurons does not completely disprove the existence of human AHN. However, it contradicts the claim that their enhanced expression levels serve as the definitive evidence of its existence and strongly indicates the overestimation of the amount of AHN, if any. Thus, we believe it is premature to conclude that AHN can truly occur in humans to the extent reported by MorenoJiménez et al. [1], Boldrini et al. [2], and Tobin et al. [3]. Moreover, we would like to draw attention to the possibility that the re-expression of the markers for cell cycle/ immature neurons may contribute to the pathophysiology of the disease and that the strategy to simply increase the expression of these markers may be ineffective or may rather worsen the symptoms.

\section{Supplementary information}

Supplementary information accompanies this paper at https://doi.org/10. 1186/s13041-019-0522-8.

Additional file 1 Table S1. Molecular evidence for the adult hippocampal neurogenesis in humans. Table S2. Literature showing evidence of the expression of stem cell/cell cycle/immature neuron markers in mature neurons.

\section{Abbreviations}

AD: Alzheimer's disease; AHN: Adult hippocampal neurogenesis; BrdU: Bromodeoxyuridine; CA1: Cornus ammonis 1; CB: Calbindin; CR: Calretinin; DCX: Doublecortin; DG: Dentate gyrus; FLX: Fluoxetine; GCL: Granule cell layer; PCNA: Proliferating cell nuclear antigen; SNAP25: Synaptosomal-associated protein 25; Sox2: SRY-box transcription factor 2 


\section{Authors' contributions}

$\mathrm{HH}$ and TMiyakawa wrote the manuscript. TMurano conducted the critical revisions of the manuscript. All authors devised the study concept and design. All authors revised the final version of the manuscript. All authors read and approved the final manuscript.

\section{Funding}

This work was supported by JSPS Grant-in-Aid for Scientific Research on Innovative Areas Grant Number JP16H06462 and AMED Strategic Research Program for Brain Sciences Grant Number JP18dm0107101.

\section{Availability of data and materials}

Not applicable.

\section{Ethics approval and consent to participate}

Not applicable.

\section{Consent for publication}

Not applicable.

\section{Competing interests}

The authors declare that they have no competing interests.

\section{Author details}

${ }^{1}$ Division of Systems Medical Science, Institute for Comprehensive Medical Science, Fujita Health University, Toyoake, Aichi 470-1192, Japan. ${ }^{2}$ Laboratory of Nutritional Brain Science, Department of Food Science and Nutrition, Mukogawa Women's University, Nishinomiya, Hyogo 663-8558, Japan.

${ }^{3}$ Cognitive Neuroscience Section, Primate Research Institute, Kyoto University, Inuyama, Aichi 484-8506, Japan.

\section{Received: 1 October 2019 Accepted: 13 November 2019} Published online: 10 December 2019

\section{References}

1. Moreno-Jiménez EP, Flor-García M, Terreros-Roncal J, Rábano A, Cafini F, Pallas-Bazarra N, et al. Adult hippocampal neurogenesis is abundant in neurologically healthy subjects and drops sharply in patients with Alzheimer's disease. Nat Med. 2019;25:554-60.

2. Boldrini M, Fulmore CA, Tartt AN, Simeon LR, Pavlova I, Poposka V, et al. Human hippocampal neurogenesis persists throughout aging. Cell Stem Cell. 2018:22:589-99 e5.

3. Tobin MK, Musaraca K, Disouky A, Shetti A, Bheri A, Honer WG, et al. Human hippocampal neurogenesis persists in aged adults and Alzheimer's disease patients. Cell Stem Cell. 2019;24:974-82 e3.

4. Herrup K, Yang Y. Cell cycle regulation in the postmitotic neuron: oxymoron or new biology? Nat Rev Neurosci. 2007:8:368-78.

5. Sharma R, Kumar D, Jha NK, Jha SK, Ambasta RK, Kumar P. Re-expression of cell cycle markers in aged neurons and muscles: whether cells should divide or die? Biochim Biophys Acta Mol basis Dis. 2017;1863:324-36.

6. Tse K-H, Herrup K. Re-imagining Alzheimer's disease - the diminishing importance of amyloid and a glimpse of what lies ahead. J Neurochem. 2017;143:432-44.

7. Kobayashi K, Ikeda Y, Sakai A, Yamasaki N, Haneda E, Miyakawa T, et al. Reversal of hippocampal neuronal maturation by serotonergic antidepressants. PNAS. 2010;107:8434-9.

8. Hagihara H, Ohira K, Miyakawa T. Transcriptomic evidence for immaturity induced by antidepressant fluoxetine in the hippocampus and prefrontal cortex. Neuropsychopharmacol Rep. 2019;39:78-89.

9. Shuto T, Kuroiwa M, Sotogaku N, Kawahara Y, Oh Y-S, Jang J-H, et al. Obligatory roles of dopamine D1 receptors in the dentate gyrus in antidepressant actions of a selective serotonin reuptake inhibitor, fluoxetine. Mol Psychiatry. 2018. https://doi.org/10.1038/s41380-018-0316-x

10. Ohira K, Hagihara H, Miwa M, Nakamura K, Miyakawa T. Fluoxetine-induced dematuration of hippocampal neurons and adult cortical neurogenesis in the common marmoset. Mol Brain. 2019;12:69.

11. Hagihara H, Takao K, Walton NM, Matsumoto M, Miyakawa T. Immature dentate gyrus: an endophenotype of neuropsychiatric disorders. Neural Plast. 2013;2013:318596.
12. Umemori J, Winkel F, Didio G, Pou ML, Castrén E. iPlasticity: induced juvenile-like plasticity in the adult brain as a mechanism of antidepressants. Psychiatry Clin Neurosci. 2018;72:633-53.

13. Shin R, Kobayashi K, Hagihara H, Kogan JH, Miyake S, Tajinda K, et al. The immature dentate gyrus represents a shared phenotype of mouse models of epilepsy and psychiatric disease. Bipolar Disord. 2013;15:405-21.

14. Imoto Y, Segi-Nishida E, Suzuki H, Kobayashi K. Rapid and stable changes in maturation-related phenotypes of the adult hippocampal neurons by electroconvulsive treatment. Mol Brain. 2017;10:8.

15. Ohira K, Kobayashi K, Toyama K, Nakamura HK, Shoji H, Takao K, et al. Synaptosomal-associated protein 25 mutation induces immaturity of the dentate granule cells of adult mice. Mol Brain. 2013;6:12.

16. Karádi K, Janszky J, Gyimesi C, Horváth Z, Lucza T, Dóczi T, et al. Correlation between calbindin expression in granule cells of the resected hippocampal dentate gyrus and verbal memory in temporal lobe epilepsy. Epilepsy Behav. 2012;25:110-9.

17. Zs M, Halász P, Vajda J, Czirják S, Freund TF. Loss of Calbindin-D28K immunoreactivity from dentate granule cells in human temporal lobe epilepsy. Neuroscience. 1997;76:377-85.

18. You JC, Muralidharan K, Park JW, Petrof I, Pyfer MS, Corbett BF, et al. Epigenetic suppression of hippocampal calbindin-D28k by $\Delta$ FosB drives seizure-related cognitive deficits. Nat Med. 2017;23:1377-83.

19. Stefanits H, Wesseling C, Kovacs GG. Loss of Calbindin immunoreactivity in the dentate gyrus distinguishes Alzheimer's disease from other neurodegenerative dementias. Neurosci Lett. 2014;566:137-41.

20. Friedman D, Honig LS, Scarmeas N. Seizures and epilepsy in Alzheimer's disease. CNS Neurosci Ther. 2012;18:285-94.

21. Murano T, Hagihara H, Tajinda K, Matsumoto M, Miyakawa T. Transcriptomic immaturity inducible by neural hyperexcitation is shared by multiple neuropsychiatric disorders. Commun Biol. 2019;2:32.

22. Malberg JE, Eisch AJ, Nestler EJ, Duman RS. Chronic antidepressant treatment increases neurogenesis in adult rat hippocampus. J Neurosci. 2000;20:9104-10.

23. Santarelli L, Saxe M, Gross C, Surget A, Battaglia F, Dulawa S, et al. Requirement of hippocampal neurogenesis for the behavioral effects of antidepressants. Science. 2003;301:805-9.

24. Yamasaki N, Maekawa M, Kobayashi K, Kajii Y, Maeda J, Soma M, et al. Alpha-CaMKII deficiency causes immature dentate gyrus, a novel candidate endophenotype of psychiatric disorders. Mol Brain. 2008;1:6.

25. Klempin F, Kronenberg G, Cheung G, Kettenmann H, Kempermann G. Properties of doublecortin-(DCX)-expressing cells in the piriform cortex compared to the neurogenic dentate gyrus of adult mice. PLoS One. 2011;6:e25760.

26. Verwer RWH, Sluiter AA, Balesar RA, Baayen JC, Noske DP, Dirven CMF, et al. Mature astrocytes in the adult human neocortex express the early neuronal marker doublecortin. Brain. 2007;130:3321-35.

27. Jessberger S, Römer B, Babu H, Kempermann G. Seizures induce proliferation and dispersion of doublecortin-positive hippocampal progenitor cells. Exp Neurol. 2005;196:342-51.

28. Spalding KL, Bergmann O, Alkass K, Bernard S, Salehpour M, Huttner HB, et al. Dynamics of hippocampal neurogenesis in adult humans. Cell. 2013; 153:1219-27.

29. Kempermann G, Gage FH, Aigner L, Song H, Curtis MA, Thuret $S$, et al. Human adult neurogenesis: evidence and remaining questions. Cell Stem Cell. 2018;23:25-30.

30. Nagy Z, Esiri MM, Smith AD. Expression of cell division markers in the hippocampus in Alzheimer's disease and other neurodegenerative conditions. Acta Neuropathol. 1997;93:294-300.

31. Perry EK, Johnson M, Ekonomou A, Perry RH, Ballard C, Attems J. Neurogenic abnormalities in Alzheimer's disease differ between stages of neurogenesis and are partly related to cholinergic pathology. Neurobiol Dis. 2012;47:155-62.

32. Jin K, Peel AL, Mao XO, Xie L, Cottrell BA, Henshall DC, et al. Increased hippocampal neurogenesis in Alzheimer's disease. PNAS. 2004;101:343-7.

33. Nagy Z, Esiri MM. Neuronal cyclin expression in the hippocampus in temporal lobe epilepsy. Exp Neurol. 1998;150:240-7.

34. Bobinski M, Wegiel J, Tarnawski M, Bobinski M, Reisberg B, de Leon MJ, et al. Relationships between regional neuronal loss and neurofibrillary changes in the hippocampal formation and duration and severity of Alzheimer disease. J Neuropathol Exp Neurol. 1997:56:414-20.

35. West MJ, Coleman PD, Flood DG, Troncoso JC. Differences in the pattern of hippocampal neuronal loss in normal ageing and Alzheimer's disease. Lancet. 1994;344:769-72. 
36. West MJ, Kawas CH, Stewart WF, Rudow GL, Troncoso JC. Hippocampal neurons in pre-clinical Alzheimer's disease. Neurobiol Aging. 2004:25:1205-12

37. Verdaguer E, García-Jordà E, Canudas AM, Domínguez E, Jiménez A, Pubill $D$, et al. Kainic acid-induced apoptosis in cerebellar granule neurons: an attempt at cell cycle re-entry. Neuroreport. 2002;13:413.

38. Negis Y, Karabay A. Expression of cell cycle proteins in cortical neuronscorrelation with glutamate-induced neurotoxicity. Biofactors. 2016;42:358-67.

39. Skene NG, Roy M, Grant SG. A genomic lifespan program that reorganises the young adult brain is targeted in schizophrenia. ELife. 2017;6:e17915.

\section{Publisher's Note}

Springer Nature remains neutral with regard to jurisdictional claims in published maps and institutional affiliations.

Ready to submit your research? Choose BMC and benefit from:

- fast, convenient online submission

- thorough peer review by experienced researchers in your field

- rapid publication on acceptance

- support for research data, including large and complex data types

- gold Open Access which fosters wider collaboration and increased citations

- maximum visibility for your research: over $100 \mathrm{M}$ website views per year

At $B M C$, research is always in progress.

Learn more biomedcentral.com/submissions 